\title{
Circumferential Deformation and Shear Stress Induce Differential Responses in Saphenous Vein Endothelium Exposed to Arterial Flow
}

\author{
Jonathan Golledge, ${ }^{\star}$ Robert J. Turner, ${ }^{\ddagger}$ Suzanne L. Harley, ${ }^{\star \ddagger}$ David R. Springall, ${ }^{\S \dagger}$ and Janet T. Powell ${ }^{\star \ddagger}$ \\ $*$ Department of Surgery and ${ }^{\ddagger}$ Department of Biochemistry, Charing Cross and Westminster Medical School, London W6 8RF, \\ United Kingdom; and ${ }^{\S}$ Department of Histochemistry, The Royal Postgraduate Medical School, London W12 0HS, United Kingdom
}

\begin{abstract}
Adaptation of saphenous vein to the hemodynamic stresses of the arterial circulation is critical to the maturation of vein bypass grafts. We have investigated early adaptive responses of venous endothelium by placing excised human saphenous vein in a bypass circuit with either venous or arterial flow conditions, using external stenting to resolve the effects of longitudinal (shear) from circumferential stress. Endothelial protein concentrations were assessed by immunostaining area (ratio of protein/CD31) and Western blotting of endothelial cell lysates (staining ratio protein/vWf).

In both unstented and stented veins nitric oxide synthase increased after $90 \mathrm{~min}$ of arterial flow: twofold increase of immunostaining area $(P=0.001)$, four- to fivefold increase by Western blotting $(P=0.02)$, and increased A23187mediated maximum endothelium-dependent relaxation of vein rings $(P=0.01)$. In unstented veins, ICAM-1 concentration was increased after 45 min of arterial flow: twofold increase by immunostaining $(P=0.001)$ and Western blotting $(P=0.038)$, with maximum fibrinogen-mediated endothelium-dependent relaxation increasing from 55.9 \pm 4.9 to $97 \pm 2.1 \%(P=0.01)$. In contrast, in unstented veins there was a threefold decrease of VCAM-1 and no change in P-selectin after arterial flow for $\mathbf{4 5}$ and $90 \mathrm{~min}$, respectively. However, no changes in ICAM-1 and VCAM-1 were observed in stented veins. The flow-induced alterations in nitric oxide synthase, ICAM-1, and VCAM-1 were abolished when $3 \mathrm{mM}$ tetraethylammonium ion ( $\mathrm{K}+$ channel blocker) was included in the vein perfusate.

The very rapid changes in ICAM-1 and VCAM-1 expression are a response to circumferential stress, whereas the slower upregulation of nitric oxide synthase is a response to longitudinal (shear) stress. Similar changes could influence the adhesiveness of endothelium in newly implanted saphenous vein bypass grafts. (J. Clin. Invest. 1997. 99:2719-2726.) Key words: nitric oxide synthase - ICAM-1 - VCAM-1 • K+ channel $\bullet$ bypass
\end{abstract}

$\dagger$ Died 27 January 1997, aged 52 years.

Address correspondence to Janet Powell, Department of Surgery, Charing Cross and Westminster Medical School, Fulham Palace Road, London W6 RF, United Kingdom. Phone: 44-181-846-7312; FAX: 44181-846-7330; E-mail: rahk700@cxwms.ac.uk

Received for publication 3 September 1996 and accepted in revised form 12 March 1997.

J. Clin. Invest.

(C) The American Society for Clinical Investigation, Inc.

0021-9738/97/06/2719/08 \$2.00

Volume 99, Number 11, June 1997, 2719-2726

\section{Introduction}

The outcome of saphenous vein bypass surgery, in both the aortocoronary and infrainguinal circulations, depends on the successful adaptation of this vessel from the low pressure venous system to the high pressure, pulsatile flow of the arterial circulation. After implantation, the vein is exposed to immediate increases in flow, longitudinal wall (shear) stress, circumferential deformation, circumferential stress, radial deformation, radial stress, pulsatile deformation, and pulsatile stress (1). The adaptation of the endothelium that occurs after implantation of a vein bypass graft is influenced by these hemodynamic forces, the endothelial injury resulting from surgical preparation, interactions between different cell types in the venous wall, and between the cellular elements of the circulation and the endothelium.

Changes in hemodynamic forces have complex effects on the endothelium. The responses of cultured endothelial cells to shear stress have been studied extensively, and much of this work has been reviewed recently (2). Immediate responses of endothelium to increased shear stress include the activation of ion channels and changes in membrane potential (3). Slower responses include changes in cell proliferation and the expression of constituitively expressed proteins. Examples of such shear stress responsive genes include those encoding PDGF, nitric oxide synthase (e-NOS), ${ }^{1}$ monocyte chemotactic protein-1 (MCP-1), and the intercellular adhesion molecule-1 (ICAM-1) (2). For some constitutively expressed genes, e.g., e-NOS, ICAM-1, and MCP-1, an increase in both transcript levels and protein expression is observed within 4-6 h of increased shear stress, with transcript levels remaining elevated for several hours before return to baseline. For other genes, e.g., the inducible endothelial-leukocyte adhesion molecule (VCAM-1), increased shear stress has the effect of reducing transcript levels over the same time period (2).

The mechanisms by which the endothelium senses and transduces changes in hemodynamic forces are poorly understood. It seems likely that flow and stretch sensitive receptors on the endothelial cell membrane, which are activated by changes in shear stress or deformation, are linked to cytoskeletal proteins and the production of second messengers. Studies in cultured endothelial cells have highlighted the importance of $\mathrm{K}+$ channels in transducing the response to increased shear stress (3-5). The nonspecific $\mathrm{K}+$ channel blocker tetraethylammonium ion (TEA) has been shown to inhibit the shear stress induced increases in e-NOS and TGF- $\beta 1$ mRNA and

1. Abbreviations used in this paper: CD31, platelet cell adhesion molecule-1; e-NOS, nitric oxide synthase; ICAM-1, intercellular adhesion molecule-1; MCP-1, monocyte chemotactic protein-1; PTFE, polytetrafluoroethylene; TEA, tetraethylammonium; VCAM-1, endothelialleukocyte adhesion molecule. 
cGMP concentrations in cultured endothelial cells. Synergistic effects of shear stress and cyclic circumferential stress also have been demonstrated, particularly with respect to the formation of stress fibers and F-actin organization in cultured endothelial cells (6). In other experiments, distinctive responses to cyclic strain and shear stress have been observed. For example, in cultured endothelial cells, sustained upregulation of MCP-1 gene expression after $1 \mathrm{~h}$ of cyclic strain has been reported, compared to only a transient response to sustained application of shear stress $(7,8)$.

The studies described above have provided valuable insight into how cultured endothelial cells respond to hemodynamic stress. However, the responses of the endothelium in vivo to hemodynamic stress may be very different from the response of cultured cells to shear stress. Recognition of the importance of these tissue responses has stimulated the recent development of techniques to study the responses of the excised vessels to flow $(9,10)$. We describe a similar technique, to simulate the implantation of a saphenous vein bypass graft, which is suitable for studying the early responses of saphenous vein endothelium to arterial flow conditions. In this technique, external stenting of vein with polytetrafluoroethylene (PTFE) can be used to resolve the effects of pulsatile longitudinal (shear) stress from pulsatile circumferential (and radial) stresses. In particular, we have investigated how arterial flow conditions influence the expression of proteins that regulate the adhesiveness of saphenous vein endothelium for leukocytes: nitric oxide synthase type III (e-NOS), P-selectin, ICAM-1, and VCAM-1.

\section{Methods}

Patients. Saphenous vein was harvested from patients undergoing aortocoronary or infrainguinal bypass, amputation, or high ligation of saphenous vein for correction of varicose veins, and immediately brought to the laboratory in ice-cold Krebs' solution. Diseased vein, which did not respond to phenylephrine $(10 \mu \mathrm{M})$ with a contraction of $>2 \mathrm{~g}$ and then relax in response to A23187 $(1 \mu \mathrm{M})$, was discarded. Samples from smokers and patients with diabetes were excluded. The use of saphenous vein for the investigations reported here was approved by the local Ethical Committee.

Materials. Fibrinogen (from either Kabi-Pharmacia, Stockholm, Sweden, or Sigma Chemical Co., St. Louis, MO) was dialyzed against Krebs' solution and the purity of the solution was assessed by SDS polyacrylamide gel electrophoresis under reducing conditions. Modified Krebs' solution ( $\mathrm{NaCl}$ : $118.4 \mathrm{mmol}$; $\mathrm{KCl}: 4.7 \mathrm{mmol}$; $\mathrm{KH}_{2} \mathrm{PO}_{4}: 1.2$ mmol; $\mathrm{MgSO}_{4} \cdot 7 \mathrm{H}_{2} \mathrm{O} 1.2 \mathrm{mmol}$; glucose: $11.1 \mathrm{mmol}$; $\mathrm{NaHCO}_{3}: 24.9$ $\mathrm{mmol}$; and $\mathrm{CaCl}_{2}: 2.5 \mathrm{mmol}$ ) was made freshly each day. Calcium ionophore (A23187), $\mathrm{N}^{\mathrm{G}}$-nitro-L-arginine methyl ester (L-NAME), glibenclamide, phenylephrine, sodium nitroprusside, TEA, collagenase for endothelial cell harvesting, biotinylated horse anti-mouse and sheep anti-rabbit antibodies, avidin, and biotin reagents were obtained from Sigma Chemical Co. Electrochemiluminescence Western blotting reagents were obtained from Amersham International (Little Chalfont, United Kingdom). Antibodies for immunohistochemistry and Western blotting were obtained as follows: ICAM-1, E- and P-selectin (monoclonal; Serotec, Kidlington Oxford, United Kingdom), e-NOS (monoclonal; kind gift of Dr. Jennifer Pollock, Department of Pharmacology and Toxicology, Medical College of Georgia, Augusta, GA; polyclonal from Santa Cruz Biotechnology, Santa Cruz, CA), VCAM-1 (monoclonal; kind gift of Professor Dorian Haskard, Department of Medicine, Royal Postgraduate Medical School, London, United Kingdom), platelet endothelial cell adhesion molecule-1 (CD31) (monoclonal; R\&D Systems Europe Ltd., Abbington, Oxon, UK) and polyclonal antibodies to vWf from DAKO (High Wycombe, Bucks, United Kingdom). MCP-1 ELISA kits were from R\&D Sys- tems Europe Ltd. Antibodies for characterization of isolated endothelial cell included vWf and CD31 (as above), anti-smooth muscle cell actin and CD45 from DAKO.

The flow circuit. Saphenous vein $(3-4 \mathrm{~cm})$ was mounted in a retaining jig after removal of a control section of vein for histology, immunohistochemistry, and organ bath studies. The jig was then placed in a bypass circuit consisting of a perfusion pump (Stockert, Munich, Germany), two water baths (one to warm the circulating Krebs' solution and one to warm the stationary solution surrounding the adventitia of the vein), the circuit apparatus, and a pressure transducer (S\&W Medico Teknik A/S, Albertslund, Denmark). The internal diameter of the vein was monitored using both B- and M-mode ultrasonography (Aloka SSD-500 with a $7.5 \mathrm{MHz}$ linear transducer), through a plastic viewing panel $1.6 \mathrm{~cm}$ above the submerged vein, at 15 -min intervals during the experiment. Veins were perfused with oxygenated Krebs' solution at $36.5^{\circ} \mathrm{C}$ (oxygen content, $20 \mathrm{ml} / \mathrm{liter}$ ). Veins were exposed either to pulsatile flow $(80 \mathrm{cpm})$ at a mean pressure of $100 \mathrm{mmHg}$ (arterial flow) for 45 or $90 \mathrm{~min}$ (flow rate, 200-225 $\mathrm{ml} / \mathrm{min}$; shear stress, $0.26 \pm 0.09 \mathrm{~N} / \mathrm{m}^{2}$ ) or to nonpulsatile flow at 20 $\mathrm{mmHg}$ (venous flow) for $90 \mathrm{~min}$ (flow rate, $10-20 \mathrm{ml} / \mathrm{min}$; shear stress, $0.021 \pm 0.011 \mathrm{~N} / \mathrm{m}^{2}$ ). The shear stress was calculated, assuming laminar flow in a cylindrical tube, by the formula:

shear stress $=32($ flow rate $\times$ fluid viscosity $) / \pi\left(\text { diameter }_{\text {mean }}\right)^{3}$.

Some veins were placed inside a tube (2-4 cm in length) of externally supported PTFE, which was nonrestrictive, but sized to limit circumferential distension of the vein during arterial flow (shear stress, $0.40 \pm 0.13 \mathrm{~N} / \mathrm{m}^{2}$ ). Diameter changes of unstented vein (mean,
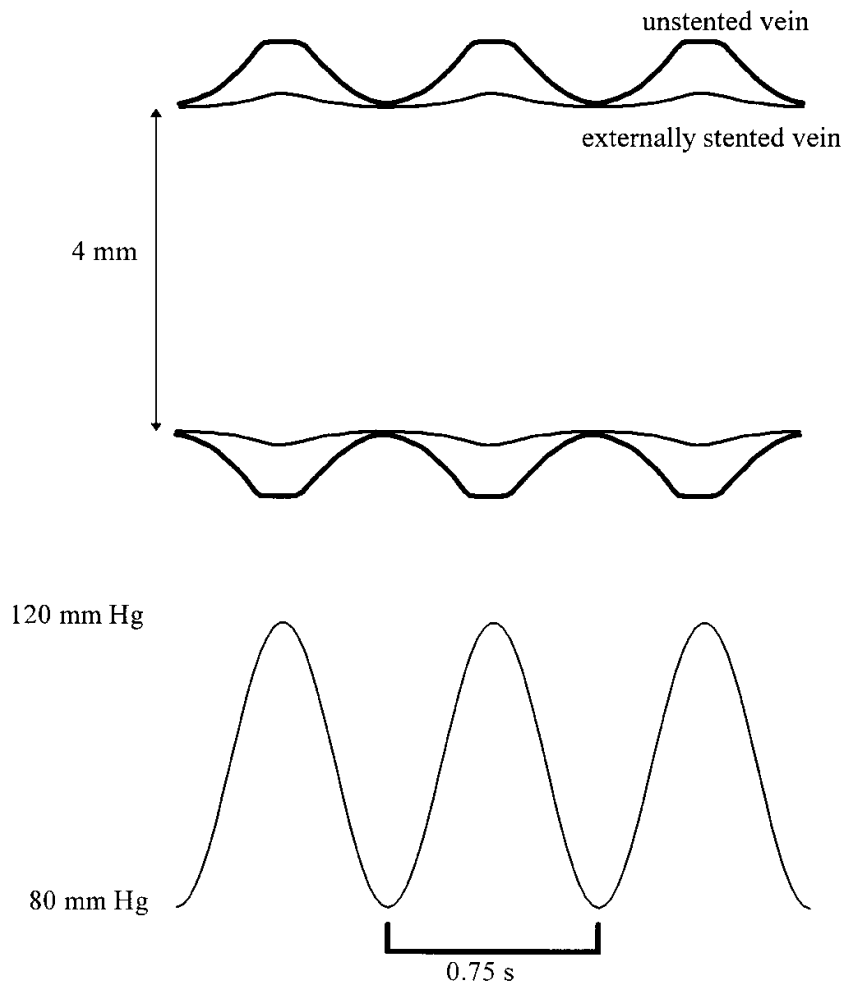

Figure 1. Cyclical changes in diameter of saphenous vein exposed to pulsatile arterial flow ex vitro. Tracings from M-mode ultrasonographic images are shown for the deformation of a vein, initial diameter $4 \mathrm{~mm}$, exposed to arterial flow for $45 \mathrm{~min}$, in two parallel circuits, one unstented and one stented; the inflow pressure waveform is given below. Very similar increases in maximum diameter (mean \pm SD, $29 \pm 4 \%$ unstented, $7 \pm 2 \%$ stented) were observed for all veins, irrespective of initial diameter (range 3.2-6.2 mm). 
$29 \pm 4 \%$ ) and stented vein (mean, $7 \pm 2 \%$ ) exposed to arterial flow conditions, with input pressure waveform, are shown in Fig. 1. In some experiments, the Krebs' solution perfusing the vein was supplemented with $3 \mathrm{mM}$ TEA. After 45 or $90 \mathrm{~min}$, the vein was removed, the ends discarded, and the remainder divided for histology, immunohistochemistry, and organ chamber studies, or used for harvesting of endothelial cells. The Krebs' solution perfusing the vein was sampled at 15-min intervals and later concentrated 10-fold through an Amicon filter with a 3,000-D exclusion limit and the concentrates were stored at $-70^{\circ} \mathrm{C}$.

Organ chamber studies. Vein rings, for assessment of endothelium-dependent relaxation, were mounted in a 10-ml organ chamber suspended between two $0.2-\mathrm{mm}$ steel wire stirrups, the upper one attached to a transducer. Responses to phenylephrine, A23187, sodium nitroprusside, and fibrinogen $(0-3 \mu \mathrm{M})$ were determined as described previously $(11,12)$.

Immunohistochemistry. Vein specimens for immunohistochemical analysis were fixed in Zamboni's solution (2\% paraformaldehyde in $0.1 \mathrm{M}$ phosphate buffer, picric acid) overnight at $4^{\circ} \mathrm{C}$, then washed in PBS containing $15 \%$ sucrose and $0.1 \%$ sodium azide. Fixed specimens were mounted on cork and frozen in isopentane suspended in liquid nitrogen. Cryostat sections $(8-10 \mu \mathrm{M})$ were prepared and serial sections stained by the ABC immunoperoxidase method (13), using monoclonal antibodies at the following dilutions: to ICAM-1 (1:800), to e-NOS $(1: 10,000)$, to VCAM-1 $(1: 20)$, to P-selectin $(1: 1,000)$, to E-selectin (up to $1: 10$ ), and to CD31 $(1: 1,000)$. The area of immunostaining was computed from serial sections for paired flow and control samples by computer-assisted image analysis (Symphony image analysis system; Seescan PLC, Cambridge, United Kingdom). Digitized images representing the whole transverse section of the vessel were segmented by interactive thresholding to separate immunostaining (endothelium) from background. The endothelial staining was highlighted using a cursor and background luminal and medial staining were excluded. The area of staining was computed from serial sections for paired flow and control samples using the same intensity setting. To minimize inconsistencies, all samples to be quantified were prepared and stained at the same time. Measurements were taken in a darkened room to minimize background illumination and the stability of the light source was assessed regularly. First, 5-10 sections taken from sites along the paired specimens ( $n=8$ pairs) were assessed for the area of CD31 staining and the mean staining area derived from averaging the results from five sections per sample. Subsequently, further serial sections (5-10 per paired sample, $n=8)$ were assessed for P-selectin, ICAM-1, e-NOS, and VCAM-1 staining area, again estimating mean staining area from five sections per sample. To allow for the small endothelial loss noted from CD31 immunostaining and the dilation that occurs in response to arterial flow, the staining areas for P-selectin, ICAM-1, e-NOS, and VCAM-1 were expressed as percentage of CD31 staining.

Western blotting. Endothelial cells were harvested from freshly excised control veins and veins exposed to arterial flow for 45 or 90 min (with or without external PTFE stenting) and also from veins exposed to arterial flow in the presence of $3 \mathrm{mM}$ TEA. The veins were opened longitudinally and the luminal surface exposed to $1 \%$ collagenase solution for $20 \mathrm{~min}$. After this time the collagenase was inactivated with RPMI containing $10 \%$ FCS, the endothelial cells were harvested by centrifugation, and then resuspended in RPMI. The yield of endothelial cells, estimated using a hemocytometer, ranged between 5,000 and 10,000 cells per vein segment. Contamination of endothelial cells by other vascular cells or leukocytes was assessed by immunostaining of cytospin preparations. Routinely preparations contained $>95 \%$ endothelial cells, $<2 \%$ leukocytes, and $<2 \%$ smooth muscle cells. Cells were collected, as a pellet, and stored at $-70^{\circ} \mathrm{C}$. The cell pellet was dispersed in lysis buffer (10 mM Tris, pH 8, 1 mM EDTA, $2.5 \%$ SDS, $5 \%$ mercaptoethanol) before SDS-PAGE (Phast System $8-25 \%$ gradient acrylamide gel). The proteins were transferred to Hybond-PVDF membrane by unidirectional diffusion and the nonspecific binding sites on the membranes were blocked according to the manufacturer's instructions. The membrane was washed with Tris-buffered saline-Tween and incubated with primary antibodies overnight (e-NOS, ICAM-1, and VCAM-1 at 1:100 dilution, and vWf at 1:1,000 dilution) before continued processing for enhanced chemiluminescence (ECL) detection. The sensitivity of the ECL technique in detecting ICAM-1 and e-NOS protein was determined using human umbilical vein endothelial cells: 1,000 cells provided adequate detection of both ICAM-1 and e-NOS. The staining intensity of specific bands was quantified by densitometric scanning. Changes in ICAM-1, VCAM-1, and e-NOS staining were standardized with respect to vWf staining. To minimize inconsistencies, paired samples always were processed together, and for proteins, where the primary antibody was monoclonal (ICAM-1 and VCAM-1), there was simultaneous development of vWf. The same batches of antibody were used throughout, except for e-NOS where a new batch of antibody was used for stented veins and veins perfused with TEA. Dot blotting and Western blotting also were used to monitor the presence of $\mathrm{vWf}$ in the vein perfusate.

Endothelial cell assay to detect upregulation of ICAM-1. Endothelial cells were isolated from freshly excised vein, as described above, and cultured in RPMI supplemented with $20 \%$ human serum and endothelial cell growth factor supplement on fibronectin-coated flasks. Confluent cells at passage 2-3 were used in 24-well plates to assay for expression of ICAM-1. Briefly, wells were incubated with concentrated vein perfusate or interleukin $1 \beta(5 \mathrm{ng} / \mathrm{ml})$, to provide positive assay standards, for $6 \mathrm{~h}$. The cells were washed and fixed with ice-cold methanol containing $0.03 \%$ hydrogen peroxide for $5 \mathrm{~min}$. The cell layers were incubated successively with antibodies to ICAM-1, peroxidase-linked second antibody, and peroxidase substrate; controls were provided by the omission of first antibody. Absorbance at $492 \mathrm{~nm}$ increased from $0.02 \pm 0.01$ in unstimulated cells to $1.40 \pm 0.02$, $6 \mathrm{~h}$ after stimulation with interleukin $1 \beta$.

Tissue ATP concentration. Samples of vein were snap frozen in a liquid nitrogen-cooled clamp. The tissue was weighed before being pulverized in liquid nitrogen and extracted into $0.9 \mathrm{M}$ perchloric acid. ATP was quantified by an enzymatic method (14).

Analysis of data. Differences in ATP concentrations were compared by Student's paired $t$ test. Immunostaining areas (mean \pm SEM) and the immunostaining of Western blots also were compared using the Student's $t$ test for paired comparisons. Relaxation of vein rings was reported as a percentage of the contraction to phenylephrine (mean \pm SEM). Evaluation of this data was performed using the Student's $t$ test for paired observations or for concentration-response curves using repeated measures analysis of variance, followed by Bonferroni multiple comparison test (Statview 4.0 for Macintosh).

\section{Results}

Assessment of vein viability. Tissue ATP concentration, vasomotor responses, histology, and immunohistochemistry were used to evaluate the viability of veins exposed to arterial flow for periods of up to $90 \mathrm{~min}$. The tissue ATP concentration in freshly excised veins was $320 \pm 11 \mathrm{nmol} / \mathrm{g}$ wet weight but increased significantly in veins exposed to arterial flow conditions for $90 \mathrm{~min}$ (Table I). Veins exposed to arterial flow for 90 min showed significantly increased contractility and sensitivity to phenylephrine as compared with freshly excised veins or veins exposed to venous flow for 90 min (Table I). In contrast, after submaximal contraction with phenylephrine, the $\mathrm{EC}_{50}$ for sodium nitroprusside-induced relaxation remained unchanged. The maximum endothelium-dependent relaxation in response to A23187 increased significantly after veins had been exposed to arterial flow conditions for $90 \mathrm{~min}$, with a significant fivefold reduction in $\mathrm{EC}_{50}$ (Table I). Hematoxylin and eosin staining of tissue sections showed well-preserved venous endothelium after exposure to arterial flow conditions for 90 
Table I. Effect of Arterial and Venous Flow Conditions on Viability and Vasomotor Function of Saphenous Vein

\begin{tabular}{lccr}
\hline & $\begin{array}{c}\text { Freshly excised } \\
\text { vein }\end{array}$ & $\begin{array}{c}\text { Venous flow } \\
\text { for 90 min }\end{array}$ & $\begin{array}{c}\text { Arterial flow } \\
\text { for 90 min }\end{array}$ \\
\hline ATP nmol/g & $320 \pm 11$ & $280 \pm 11$ & $566 \pm 50^{*}$ \\
CD31 staining area mm ${ }^{2}$ & $0.73 \pm 0.08$ & $0.77 \pm 0.08$ & $0.65 \pm 0.08$ \\
Contraction to phenylephrine & & & \\
$\quad C_{50}(\mu M)$ & $10 \pm 2$ & $9 \pm 1$ & $1 \pm 0.4^{*}$ \\
$\quad$ Maximum (g) & $2.5 \pm 0.3$ & $3.0 \pm 0.4$ & $5.0 \pm 0.4^{*}$ \\
Relaxation to A23187 & & & \\
$\quad$ EC 5 (nM) & $46 \pm 9$ & $30 \pm 3$ & $9 \pm 1^{*}$ \\
$\quad$ Maximum (percentage) & $50 \pm 3$ & $54.4 \pm 7$ & $68.6 \pm 3^{*}$ \\
Relaxation to fibrinogen & & & \\
$\quad$ EC $C_{50}$ (nM) & $870 \pm 80$ & $860 \pm 70$ & $859 \pm 70$ \\
$\quad$ Maximum (percentage) & $61 \pm 4.9$ & $55 \pm 4.9$ & $97 \pm 2.1^{*}$ \\
& & &
\end{tabular}

${ }^{*} P<0.05$ in comparison with freshly excised vein and venous flow. Values are given as mean \pm SEM.

min; immunohistochemical staining for CD31 provided more quantitative results. Handling of freshly excised vein resulted in minor endothelial loss, demonstrated as a reduction in CD31 staining area from the expected maximum of $100 \%$ to $\sim 95 \%$; results for vWf were similar. The percentage of CD31 staining area was unaffected by venous flow circuits, but was reduced by a further 5-10\% after exposure to arterial flow circuits (Table I). The concentration of vWf in the concentrated (10 times) vein perfusate collected after 90 min of arterial flow was $<25 \mathrm{pg} / \mathrm{ml}$. Together these different forms of assessment provided reassurance about the maintenance of vein viability for periods of up to $90 \mathrm{~min}$ in the flow circuit.

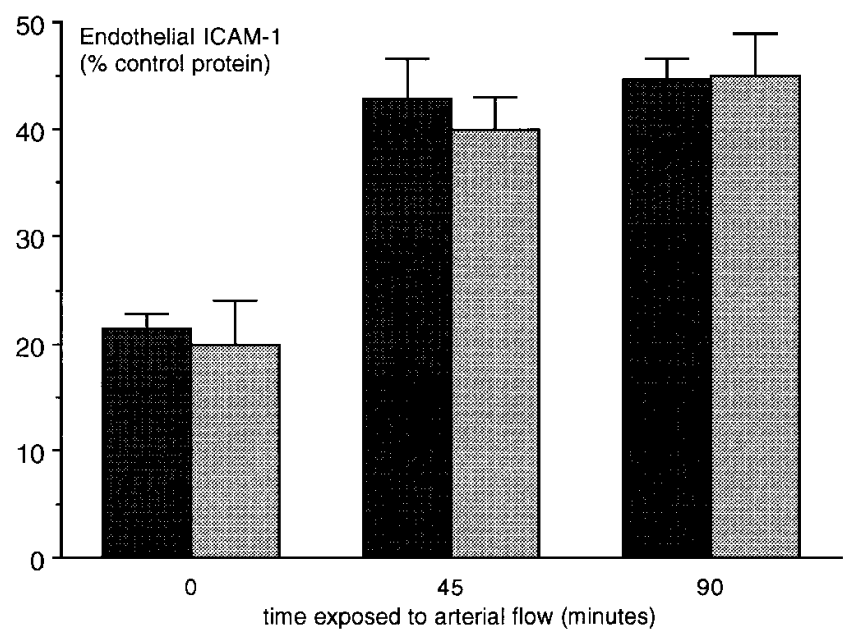

Figure 3. ICAM-1 on saphenous vein endothelium increases in response to arterial flow. Comparison of immunostaining and Western blotting experiments. Mean results from Western blotting (gray bars, ratio of ICAM-1 to vWf) and from immunostaining (dark bars, ratio of ICAM-1 to CD31) are shown. Error bars show SEM.

Expression of ICAM-1 on saphenous vein endothelium: influence of pulsatile arterial flow. There was scanty immunostaining for ICAM-1 on the endothelium of freshly excised veins or veins exposed to venous flow conditions for $90 \mathrm{~min}$, but after exposure to arterial flow for 45 or 90 min there was increased staining (Fig. 2), with a significant 1.5-2-fold increase in the ratio of ICAM-1/CD31 staining area (Fig. 3). No changes in ICAM-1 staining were observed after exposure to

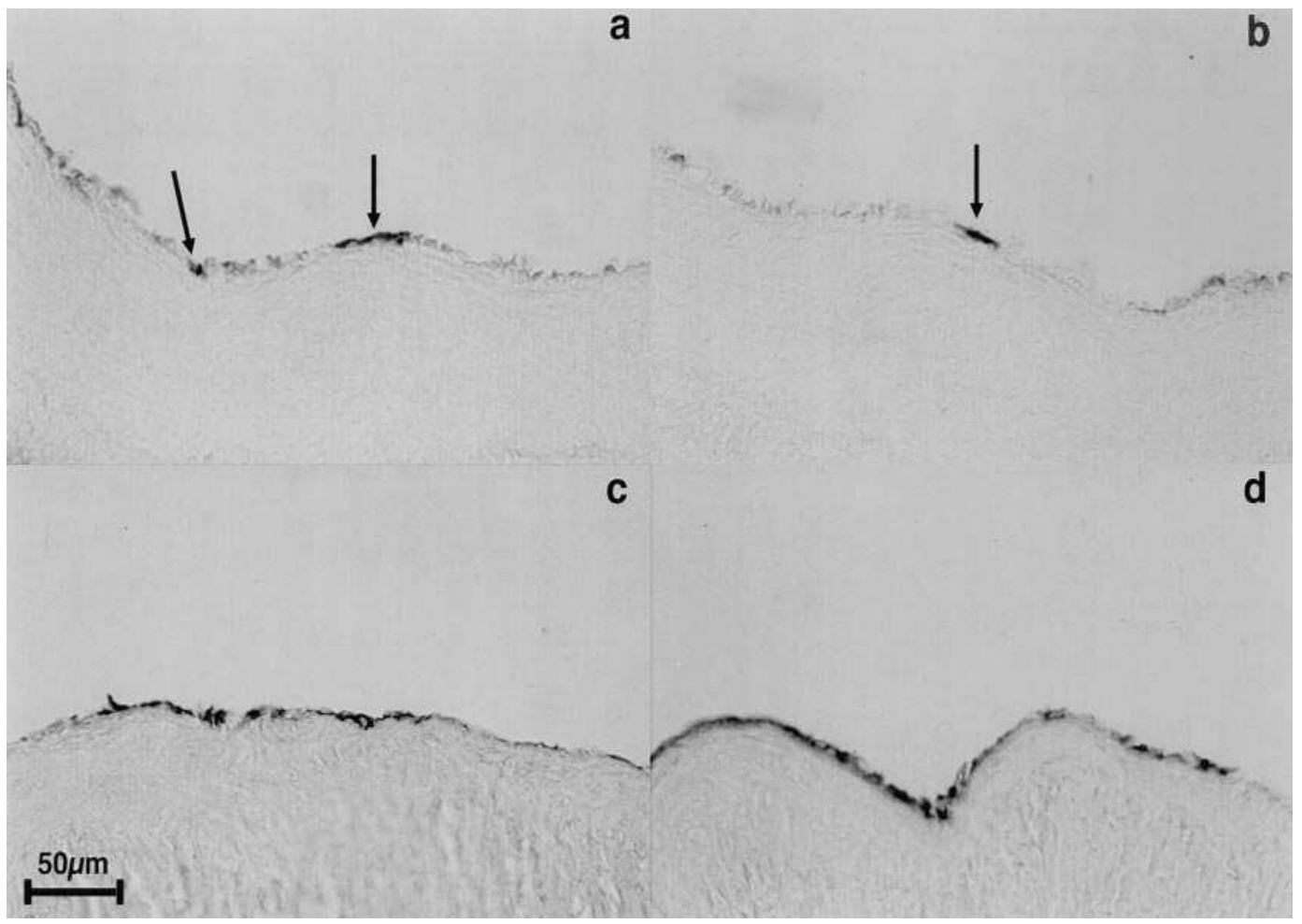

Figure 2. ICAM-1 on saphenous vein endothelium. (a) Freshly excised vein; $(b)$ vein exposed to venous flow for $90 \mathrm{~min}$; (c) unstented vein exposed to arterial flow for $45 \mathrm{~min}$; and $(d)$ unstented vein exposed to arterial flow for $90 \mathrm{~min}$. The arrows indicate the patchy staining on freshly excised vein and vein exposed to venous flow. The scale bar represents $50 \mu \mathrm{m}$. 


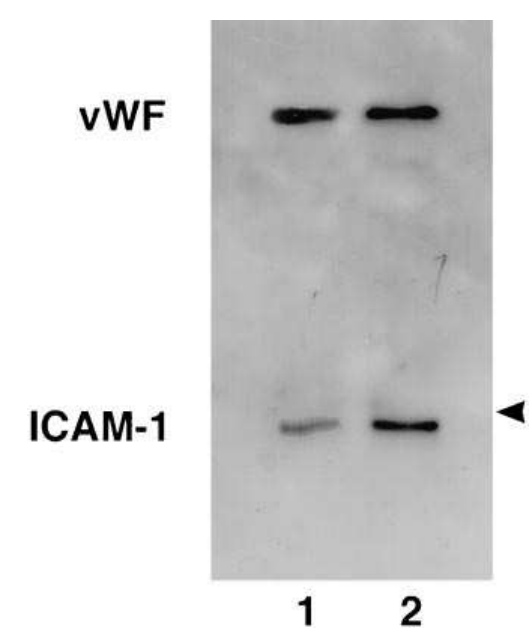

Figure 4. Detection of ICAM-1 in endothelial cell lysates by Western blotting. Simultaneous Western blotting for ICAM-1 and vWf was performed. Lane 1, endothelial lysates from freshly excised vein; lane 2, endothelial cell lysates from vein exposed to arterial flow for $90 \mathrm{~min}$, where there is clear evidence of the increased staining for ICAM-1. Arrowhead, 97-kD molecular weight calibration.

venous flow circuits for $90 \mathrm{~min}$. These changes were confirmed by Western blotting: the increased staining for ICAM-1 on lysates of endothelial cells obtained after vein had been exposed to arterial flow for $90 \mathrm{~min}$ is shown in Fig. 4. The staining ratio ICAM-1/vWf increased from $0.20 \pm 0.04$ in lysates of cells from freshly excised vein to $0.40 \pm 0.03$ (six paired samples, $P=$ 0.038 ) and $0.45 \pm 0.04$ (six paired samples, $P=0.035$ ) in lysates of cells harvested after vein had been exposed to arterial flow for 45 and $90 \mathrm{~min}$, respectively. The temporal changes in ICAM-1 are summarized in Fig. 3. The endothelium-dependent relaxation of vein rings in response to fibrinogen is probably mediated by an ICAM-1-dependent pathway (12). Maximum endothelial-dependent relaxation in response to fibrinogen increased from $61 \pm 4.9 \%$ in freshly excised veins to $90 \pm 5.4 \%$ $(P=0.01)$ and $97 \pm 2.1 \%(P<0.01$ in veins exposed to arterial flow for $45(n=8)$ and $90 \mathrm{~min}(n=10)$, respectively. Fibrinogen-mediated relaxation was unaffected by venous flow for 90 min (six paired samples, mean maximum relaxation 55 44.9 and $61 \pm 1.3 \%$, respectively). Responses to fibrinogen were abolished if vein rings were preincubated with $25 \mu \mathrm{M}$ glibenclamide. Since we detected no important differences between freshly excised vein and vein exposed to venous flow conditions for $90 \mathrm{~min}$ in the circuit, all further experiments, described in the sections below, used freshly excised vein as the control for veins exposed to the flow circuit.

We considered it important to investigate the possibility that the rapid upregulation of ICAM- 1 observed on saphenous vein endothelium in response to pulsatile arterial flow was mediated by soluble factors secreted into the vein perfusate. Cultured human saphenous vein endothelial cells express low levels of ICAM-1; the eightfold upregulation of ICAM-1 over $6 \mathrm{~h}$ in response to interleukin $1 \beta$ was monitored by a cell-based ELISA assay. Incubation of cultured human saphenous vein endothelial cells with either undiluted or concentrated (10 times) vein perfusate did not impair endothelial cell viability or effect any increased immunostaining for ICAM-1 over a period of up to $6 \mathrm{~h}$. Therefore, it appears unlikely that the rapid upregulation of ICAM-1 in response to arterial flow conditions is mediated by soluble factors. Additionally, no MCP-1 could be detected in the concentrated perfusate sampled after $90 \mathrm{~min}$, with an assay detection limit of $30 \mathrm{pg} / \mathrm{ml}$.

Expression of P-selectin, VCAM-1, and e-NOS in saphenous vein endothelium: influence of pulsatile arterial flow. After

Table II. ICAM-1, e-NOS, VCAM-1, and P-selectin on Saphenous Vein: Effect of Arterial Flow on Endothelial Immunostaining Area

\begin{tabular}{lcccc}
\hline Flow conditions & ICAM-1 & e-NOS & VCAM-1 & P-selectin \\
\hline $\begin{array}{l}\text { Freshly excised vein } \\
\text { Unstented arterial } \\
\quad \text { flow }(90 \text { min) }\end{array}$ & $21.4 \pm 1.4$ & $50.0 \pm 5.6$ & $16.6 \pm 3.4$ & $48.0 \pm 2.0$ \\
$\begin{array}{l}\text { Stented arterial flow } \\
\quad(90 \text { min) }\end{array}$ & $24.0 \pm 3.0$ & $73.7 \pm 5.0^{*}$ & $17.0 \pm 2.0$ & $49.5 \pm 2.8$ \\
$\begin{array}{l}\text { Arterial with 3 mM } \\
\text { TEA (90 min) }\end{array}$ & $20.0 \pm 1.0$ & $47.0 \pm 3.0$ & $17.5 \pm 2.0$ & ND \\
\hline
\end{tabular}

Values are mean \pm SEM of staining area for protein/CD31 (percentage). $* P \leq 0.01$ in comparison with freshly excised vein. $N D$, not determined.

exposure to arterial flow conditions for 45 or $90 \mathrm{~min}$, both the staining area ratio of P-selectin/CD31 and the P-selectin/vWf ratio observed by Western blotting remained unchanged. The staining area ratio of VCAM-1/CD31 decreased threefold after 45 or $90 \mathrm{~min}$ of exposure to arterial flow (each eight paired samples, $P=0.01$ and $P<0.01$, respectively) (Table II). These changes in VCAM-1 were confirmed by Western blotting where a fourfold decrease in VCAM-1/vWf staining ratio was observed after 90 min of arterial flow conditions (Table III). Using a monoclonal antibody to e-NOS the staining area ratio for e-NOS/CD31 increased only 1.2-fold after 45 min of arterial flow conditions, but had increased 1.6-fold (eight paired samples, $P=0.01$ ) after $90 \mathrm{~min}$ of arterial flow conditions (Fig. 5 , Table II). These changes in e-NOS were more marked on Western blotting using a polyclonal antibody, where the ratio of e-NOS/vWf staining was $0.3 \pm 0.04,0.5 \pm 0.1$, and $1.5 \pm 0.1$ $(P=0.02)$ in lysates of endothelial cells isolated from freshly excised veins and veins exposed to arterial flow conditions for $45(n=5)$ and $90 \min (n=6)$, respectively (Table III). The early changes in the expression of ICAM-1, P-selectin, VCAM-1, and e-NOS in response to arterial flow conditions are summarized in Tables II and III.

Additionally, production of nitric oxide was assessed from the endothelium-dependent relaxation of vein rings in response to increasing concentrations of A23187, as described above. There were significant increases in mean maximum relaxation (at $1 \mu \mathrm{M}$ A23187) after both 45 and 90 min of expo-

Table III. Concentration of ICAM-1, e-NOS, VCAM-1, and $P$-selectin in Endothelial Cell Lysates Assessed by Western Blotting

\begin{tabular}{lcccc}
\hline Flow conditions & ICAM-1 & e-NOS & VCAM-1 & P-selectin \\
\hline $\begin{array}{l}\text { Freshly excised vein } \\
\text { Unstented arterial }\end{array}$ & $0.20 \pm 0.04$ & $0.30 \pm 0.04$ & $0.38 \pm 0.06$ & $0.35 \pm 0.07$ \\
$\quad$ flow (90 min) & $0.45 \pm 0.04 *$ & $1.5 \pm 0.01^{*}$ & $0.13 \pm 0.04 *$ & $0.34 \pm 0.05$ \\
$\begin{array}{c}\text { Stented arterial flow } \\
\quad(90 \text { min })\end{array}$ & $0.19 \pm 0.03$ & $0.60 \pm 0.06^{*}$ & ND & ND \\
$\begin{array}{c}\text { Arterial with 3 mM } \\
\text { TEA (90 min) }\end{array}$ & $0.23 \pm 0.04$ & $0.25 \pm 0.05$ & ND & ND \\
\end{tabular}

Values are mean \pm SEM ratio of staining density for protein/vWf. $* P<$ 0.05 in comparison with freshly excised vein. $N D$, not determined. 


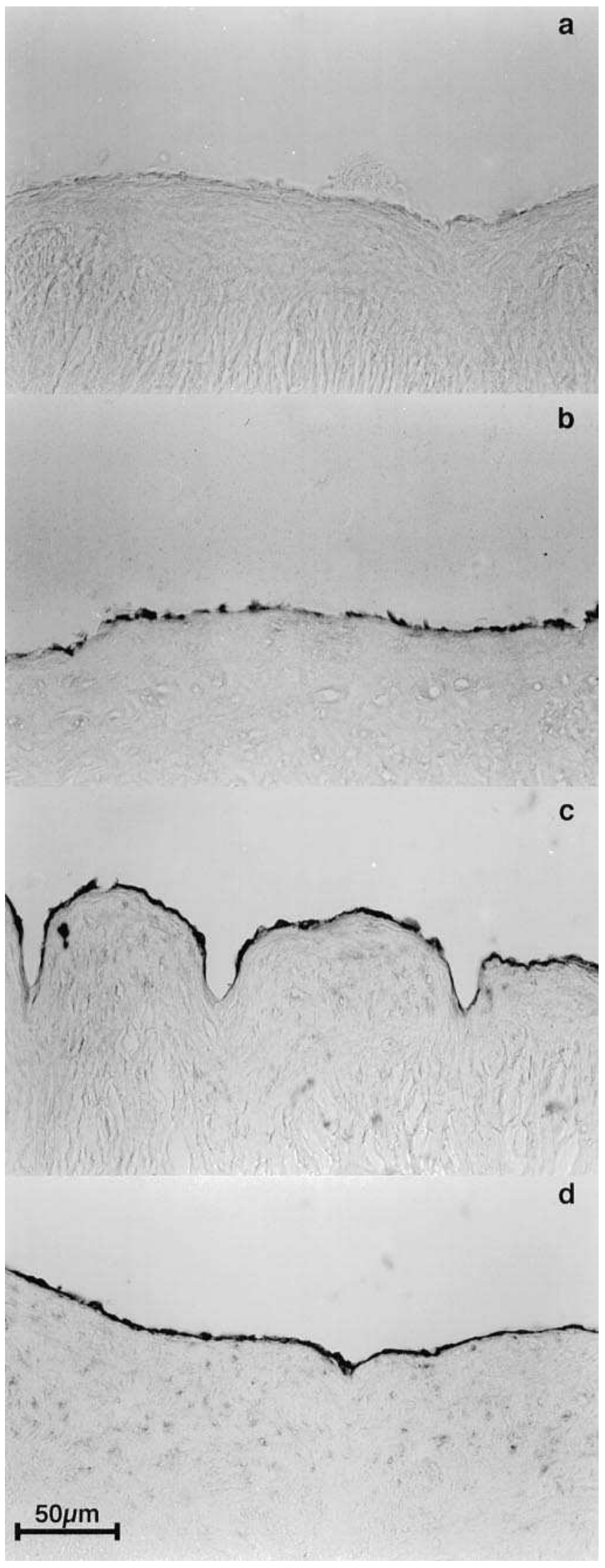

Figure 5. Nitric oxide synthase on saphenous vein endothelium. (a) Staining control with no primary antibody; $(b)$ freshly excised vein; (c) unstented vein exposed to arterial flow for $45 \mathrm{~min}$; and $(d)$ unstented vein exposed to arterial flow for $90 \mathrm{~min}$. The scale bar represents $50 \mu \mathrm{m}$. sure to arterial flow conditions, from $50 \pm 3.0$ to $67.5 \pm 3.1 \%$ (seven paired samples, $P=0.02$ ) and $68 \pm 4.6 \%$ (seven paired samples, $P=0.01$ ), accompanied by a significant fivefold reduction in $\mathrm{EC}_{50}$ (Table I). For every flow condition, preincubation of vein rings with $0.1 \mathrm{mM}$ L-NAME inhibited the relaxation in response to $\mathrm{A} 23187$.

None of the flow conditions yielded vein where positive endothelial staining for E-selectin or PDGF was observed in the Zamboni-fixed material.

Limitation of circumferential stresses in externally stented veins: alteration of endothelial response to flow. Nonrestrictive stenting with PTFE tubes limits both circumferential and radial stress (Fig. 1), hence increasing longitudinal (shear) stress from $0.26 \pm 0.09$ to $0.40 \pm 0.13 \mathrm{~N} / \mathrm{m}^{2}$ under arterial flow conditions. After $90 \mathrm{~min}$ of exposure to arterial flow conditions there was no increase in the immunostaining area ratios for either ICAM-1/CD31 or VCAM-1/CD31 (Table II). Similarly, the lysates of cells isolated from these stented veins exposed to arterial flow showed no alteration in ICAM-1 staining on Western blotting, compared with freshly excised vein (Table III).

However, the immunostaining area ratio for e-NOS/CD31 increased twofold in externally stented veins exposed to arterial flow conditions for $90 \mathrm{~min}$, eight paired samples, $P=0.001$ (Table III). Western blotting also indicated a twofold increase in the e-NOS/vWf staining area ratio in stented veins exposed to arterial flow conditions for $90 \mathrm{~min}$ (five paired samples, $P=0.03$ ) (Table III). Further, external stenting of veins was associated with the largest changes in endothelium-dependent relaxation in response to A23187, with maximum relaxation increasing from $54 \pm 7.4 \%$ in freshly excised veins to $80 \pm 4 \%$ after 90 min of arterial flow conditions (seven paired samples, $P=0.01$ ) (Fig. $6 a$ ). In contrast, there was no alteration of the vasomotor response to fibrinogen (Fig. $6 b$ ).

Potassium channel blockade: limitation of flow responses. When $3 \mathrm{mM}$ TEA was included in the vein perfusate, no significant changes were observed in the immunostaining area ratios (protein/CD31) for P-selectin, ICAM-1, VCAM, or e-NOS on veins exposed to arterial flow conditions for $90 \mathrm{~min}$ compared with freshly excised vein controls (seven paired samples) (Table II). Similarly, there were no changes of the staining ratio (ICAM-1/vWf, e-NOS/vWf) in Western blotting experiments (Table III). Supplementation of the vein perfusate with TEA also abolished the arterial flow-induced alterations in the endothelium-dependent vasomotor responses to A23187 and fibrinogen (Fig. 6, $a$ and $b$ ) even after thorough washing of vein rings with oxygenated Krebs' solution.

\section{Discussion}

The changes in endothelial protein expression that occur when saphenous vein is exposed to arterial flow conditions are similar to, but occur with greater rapidity than, the changes described when cultured endothelial cells are exposed to shear stress or cyclic strain. For example, on saphenous vein endothelium the surface expression of ICAM-1 increases twofold, while the surface expression of VCAM-1 decreases threefold within $45 \mathrm{~min}$ of exposure to arterial flow conditions. The rapidity of these responses appears to be attributable largely to the increased circumferential (and radial) deformation of veins exposed to pulsatile arterial flow, since limitation of the circumferential deformation by nonrestrictive external stenting abolished the rapid changes in surface expression of both 


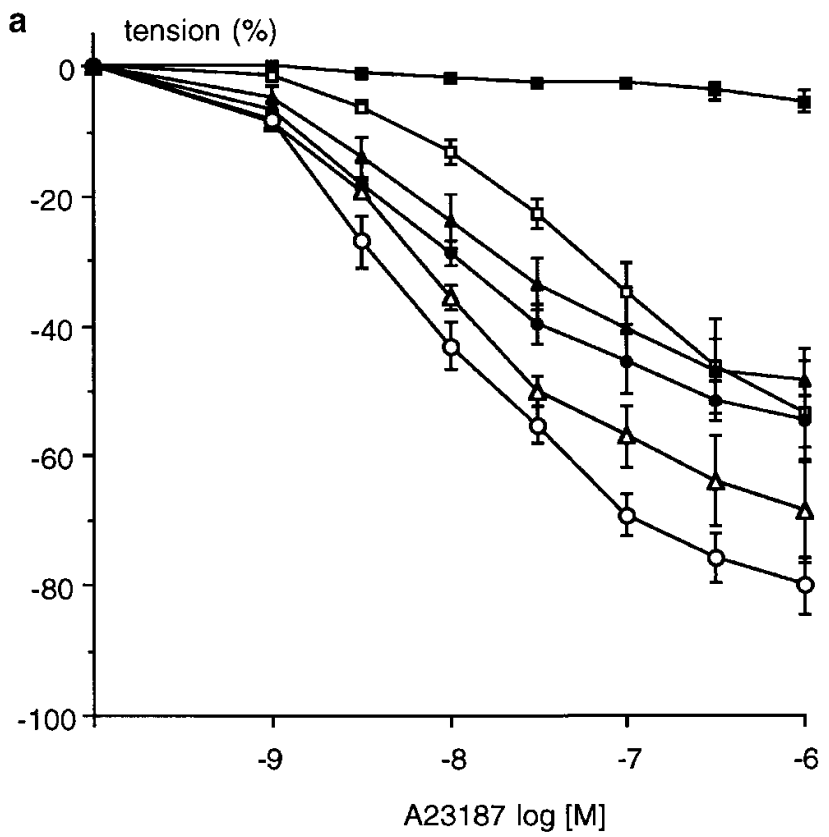

b

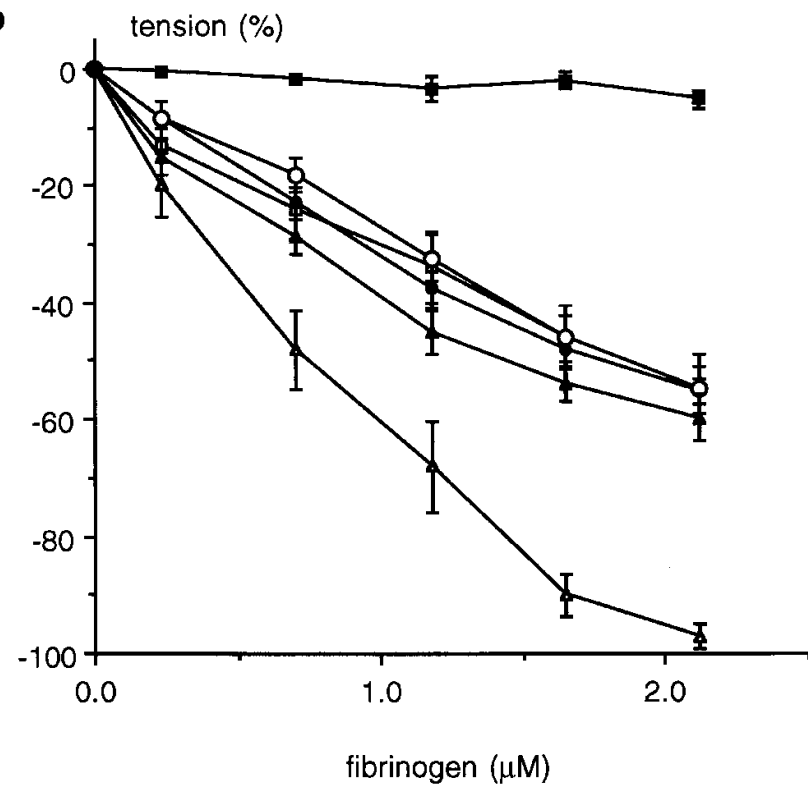

Figure 6. Endothelium-dependent relaxation of vein rings in response to the calcium ionophore A23187 and fibrinogen. (a) The responses to A23187 of freshly excised veins (open square), veins exposed to venous flow for 90 min (filled circle), and veins exposed to arterial flow for $90 \mathrm{~min}$ in the presence of $3 \mathrm{mM}$ TEA (filled triangle) were similar to those reported previously (11), whereas both stented (open circle) and unstented (open triangle) veins exposed to arterial flow for 90 min exhibited increased maximal relaxation $(1 \mu \mathrm{M}$ A23187), paired $t$ test $P=0.01$, for both stented and unstented vein. Relaxation was inhibited by preincubation of vein rings with $0.1 \mathrm{mM}$ L-NAME (filled square). (b) The responses to fibrinogen of freshly excised veins (open square), veins exposed to venous flow for $90 \mathrm{~min}$ (filled circle), and stented veins exposed to arterial flow for $90 \mathrm{~min}$ (open circle) were similar to those reported previously (12). Un-
ICAM-1 and VCAM-1. In contrast, the slower doubling in the concentration of endothelial nitric oxide synthase appears to be principally a response to increased longitudinal or shear stress, the response being maintained in externally stented veins. Other proteins, e.g., P-selectin and CD31, remained unaltered by arterial flow conditions. These findings imply the presence of separate mechanosensors and signaling pathways for shear stress and circumferential deformation on saphenous vein endothelium. These pathways appear linked to $\mathrm{K}+$ channels downstream, since TEA abolishes the arterial flowinduced endothelial changes in ICAM-1, VCAM-1, and nitric oxide synthase concentrations.

For cultured endothelial cells, the fastest documented shear stress-induced change in protein expression is a fivefold increase in c-fos within $1 \mathrm{~h}$ (15). For nitric oxide synthase, a two- to threefold increase in protein expression has been demonstrated after $6 \mathrm{~h}$ of shear stress in cultured endothelial cells of both arterial and venous origin, although it was implied that these changes may be observed as early as $3 \mathrm{~h}$ after imposition of shear stress (16). In cultured human umbilical vein endothelial cells, a two- to threefold increase in ICAM-1 protein expression was not observed until $6-8 \mathrm{~h}$ after imposition of shear stress $(17,18)$. The effects of shear stress on endothelial expression of VCAM-1 appear inconsistent, with different studies showing either unchanged or decreased surface expression $(2,17,18)$.

Many other studies have ignored protein expression and focused on changes in gene expression. We used both immunostaining area and Western blotting to quantitate protein expression. For every condition investigated, the results of immunostaining area paralleled the results obtained from Western blotting. For ICAM-1 and VCAM-1, where the same antibody was used for immunostaining and Western blotting, the techniques showed quantitatively similar changes. For nitric oxide synthase, where both different antibodies and batches were used, Western blotting with a polyclonal antibody indicated greater increases in protein concentration than the immunostaining with a monoclonal antibody. Functional assays of endothelium-dependent relaxation provided further confirmation of the altered activity of nitric oxide synthase and concentration of ICAM-1: endothelium-dependent relaxation of vein rings in response to $\mathrm{A} 23187$ or fibrinogen were used as functional indicators of nitric oxide production and ICAM-1 expression, respectively (12).

Since completion of ex vivo experiments took $2-3 \mathrm{~h}$ we were concerned about monitoring tissue viability. Three experimental findings suggested that the vein could be kept viable for this time period in oxygenated Krebs' solution. First, tissue ATP content increased after exposure of vein to arterial flow, perhaps an early sign of muscle adaptation to increased work. Second, both the contractility and endothelium-dependent vasorelaxation of vein rings was preserved or even enhanced after exposure of tissue to venous or arterial flow con-

stented veins exposed to arterial flow for $90 \mathrm{~min}$ (open triangle) exhibited enhanced maximum relaxation, paired $t$ test $P<0.01$, which was blocked by preincubation of vein rings with $5 \mu \mathrm{M}$ glibenclamide (filled square). Unstented veins exposed to arterial flow for $90 \mathrm{~min}$ in the presence of $3 \mathrm{mM}$ TEA (filled triangle) failed to show enhanced maximum relaxation despite thorough washing of the vein rings with oxygenated Krebs' solution. 
ditions for $90 \mathrm{~min}$, respectively. Third, morphological integrity of endothelium was demonstrated using both routine histological staining and immunostaining for CD31.

Nonrestrictive external stenting of veins with PTFE limited the circumferential (radial) deformation, but provided higher longitudinal shear stresses than in unstented veins. In stented veins exposed to arterial flow, no changes were observed in the concentrations of ICAM-1 or VCAM- 1 by either immunostaining or Western blotting, and the exaggerated fibrinogenmediated relaxation observed in unstented veins was abolished. Hence, the rapid increases in endothelial ICAM-1 and decreases in VCAM-1 concentration appear to be attributable to pulsatile circumferential deformation in unstented veins. In contrast, the increases in nitric oxide synthase concentration and exaggerated endothelium-dependent relaxation to A23187 were observed in stented veins after $90 \mathrm{~min}$ of arterial flow. This increase in immunostaining area for nitric oxide synthase was similar in unstented and stented veins, indicating that this was a response to increased shear stress. However, Western blotting experiments suggested a much greater increase in nitric oxide synthase concentration in unstented veins compared with stented veins. This disparity could result either from different batches of antibody being used for unstented and stented veins, or the Western blotting could indicate some synergy between circumferential deformation and shear stress to effect increases in nitric oxide synthase concentration. The latter explanation would agree with the findings that cyclic strain upregulates nitric oxide synthase in cultured arterial endothelial cells, albeit much more slowly (19).

Problems resulting from the abrupt adaptation of saphenous vein to the arterial circulation may be unique to cardiovascular surgeons. However, each year thousands of patients undergo aortocoronary or infrainguinal vein bypass surgery, with $\sim 30 \%$ of these grafts failing or needing revision within the first year after implantation. Many of these graft failures are attributed to the development of focal stenoses caused by intimal hyperplasia. Recently, experimental studies have indicated that external stenting of vein grafts can reduce significantly the development of intimal hyperplasia (20). This highlights the importance of understanding how saphenous vein grafts respond to circumferential deformation. Our study has shown that, in saphenous vein exposed to pulsatile arterial flow conditions, it is circumferential deformation, rather than shear stress, that induces the rapid changes in endothelial expression of ICAM-1 and VCAM-1, whereas the increase in nitric oxide synthase concentration appears to be principally a response to increased shear stress.

\section{Acknowledgments}

We thank Professors R.M. Greenhalgh and K.M. Taylor for their encouragement.
This work was supported by the Charing Cross Hospital Trustees, the Medical Research Council (G94-31330), and the British Heart Foundation (96/062).

\section{References}

1. Dobrin, P.B., F.N. Littooy, and E.D. Endean. 1989. Mechanical factors predisposing to intimal hyperplasia and medial thickening in autogenous vein grafts. Surgery (St. Louis). 105:393-400.

2. Resnick, N., and M.A.J. Gimbrone. 1995. Hemodynamic forces are complex regulators of endothelial gene expression. FASEB (Fed. Am. Soc. Exp. Biol.) J. 9:874-882.

3. Ohno, M., G.H. Gibbons, V.J. Dzau, and J.P. Cooke. 1993. Shear stress elevates endothelial cGMP. Role of a potassium channel and G protein coupling. Circulation. 88:193-197.

4. Ohno, M., J.P. Cooke, V.J. Dzau, and G.H. Gibbons. 1995. Fluid shear stress induces endothelial transforming growth factor beta-1 transcription and production. J. Clin. Invest. 95:1363-1369.

5. Tsao, P.S., N.P. Lewis, S. Alpert, and J.P. Cooke. 1995. Exposure to shear stress alters endothelial adhesiveness. Circulation. 92:3513-3519.

6. Zhao, S., A. Suciu, T. Ziegler, J.E. Moore, Jr., E. Burki, J.J. Meister, and H.R. Brunner. 1995. Synergistic effects of fluid shear stress and cyclic circumferential stretch on vascular endothelial cell morphology and cytoskeleton. Arterioscler. Thromb. Vasc. Biol. 15:1781-1786.

7. Shyy, Y.-J., H.J. Hsieh, S. Usami, and S. Chien. 1993. Fluid shear stress induces a biphasic response of human monocyte chemotactic protein 1 gene expression in vascular endothelium. Proc. Natl. Acad. Sci. USA. 91:4678-4682.

8. Wang, D.L., B.-S. Wung, Y.-J. Shyy, C.-F. Lin, Y.-J. Chao, S. Usami, and S. Chien. 1995. Mechanical strain induces monocyte chemotactic protein-1 expression in endothelial cells. Circ. Res. 77:294-302.

9. Schwartz, L.B., C.M. Purut, M.F. Massey, J.C. Pence, P.K. Smith, and R.L. McCann. 1996. Effects of pulsatile perfusion on human saphenous vein vasoreactivity: a preliminary report. Cardiovasc. Surg. 4:143-149.

10. Labadie, R.F., J.F. Antaki, J.L. Williams, S. Katyal, J. Ligush, S.C. Watkins, S.I.M. Pham, and H.S. Borovetz. 1996. Pulsatile perfusion system for ex vivo investigation of biochemical pathways in intact vascular tissue. Am. J. Physiol. 270:H760-H768.

11. Higman, D.J., A.M.J. Strachan, L. Buttery, R.C.J. Hicks, D.R. Springall, R.M. Greenhalgh, and J.T. Powell. 1996. Smoking impairs the activity of endothelial nitric oxide synthase in saphenous vein. Arterioscler. Thromb. Vasc. Biol. 16:546-552.

12. Hicks, R.C.J., J. Golledge, R. Mir-Hasseine, and J.T. Powell. 1996. Vasoactive effects of fibrinogen on saphenous vein. Nature (Lond.). 379:818-820.

13. Springall, D.R., V. Riveros-Moreno, L. Buttery, A. Suburo, A.E. Bishop, M. Merrett, S. Moncada, and J.M. Polak. 1992. Immunological detection of nitric oxide synthase in human tissue using heterologous antibodies suggesting different isoforms. Histochemistry. 98:259-266.

14. Lamprecht, W., and I. Trauschold. 1963. Determination of adenosine5'-triphosphate. In Bergmeyer's Methods of Enzymatic Analysis. 2101-2109.

15. Ranjan, V., and S.L. Diamond. 1993. Fluid shear stress induces synthesis and nuclear localization of c-fos in cultured human endothelial cells. Biochem. Biophys. Res. Commun. 196:79-84.

16. Ranjan, V., Z. Xiao, and S.L. Diamond. 1995. Constitutive NOS expression in cultured endothelial cells is elevated by fluid shear stress. Am. J. Physiol. 269:H550-H555.

17. Nagel, T., N. Resnick, W.J. Atkinson, C.F. Dewey, and M.A. Gimbrone. 1994. Shear stress selectively upregulates intercellular adhesion molecule-1 expression in cultured vascular endothelial cells. J. Clin. Invest. 94:885-891.

18. Morigi, M., C. Zoja, M. Figliuzzi, M. Foppolo, G. Micheletti, M. Bontempelli, M. Saronni, G. Remuzzi, and A. Remuzzi. 1995. Fluid shear stress modulates surface expression of adhesion molecules by endothelial cells. Blood. 85:1696-1703.

19. Alowesi, M.A., W.C. Sessa, and B.E. Sumpio. 1995. Cyclic strain upregulates nitric oxide synthase in cultured endothelial cells. J. Clin. Invest. 96:1449-1454.

20. Izzat, M.B., D. Mehta, A.J. Bryan, B. Reeves, A.C. Newby, and G.D. Angelini. 1996. Influence of external stent size on early medial and neointimal thickening in a pig model of saphenous vein bypass grafting. Circulation. 94: 1741-1745. 